\title{
On numerical solution of fractional order delay differential equation using Chebyshev collocation method
}

\author{
Ajmal Ali and Norhashidah Hj Mohd Ali \\ School of Mathematical Science, Universiti Sains Malaysia, 11800 Penang, Malaysia
}

Received: 19 July 2017, Accepted: 9 October 2017

Published online: 28 December 2017.

\begin{abstract}
The main objective of this article is to present an efficient numerical method to solve the delay differential equation of fractional order. We use the Caputo 's fractional derivative for solving the fractional differentiation. The properties of shifted Chebyshev polynomials are exploited to reduce the Delay Fractional Differential Equation (DFDE) to a linear or non-linear easily solvable system of algebraic equations. A comparison is given between the present method and Adomian Decomposition Method (ADM) with the help of solved numerical illustrative example. The results shows that proposed method is very effective and simple. Which reveals the validity and applicability of the method.
\end{abstract}

Keywords: Chebyshev polynomials, Caputo fractional derivative, fractional delay differential equation.

\section{Introduction}

In recent years, fractional calculus has become more important than the ordinary calculus. The ordinary calculus has achieved the discovery at its peak point. That's why, the mathematician and researchers feel the need of fractional calculus. Fractional Differential Equations (FDEs) is generalization of Ordinary Differential Equations (ODEs) because FDEs describe values on each point continually and distinguished the gaps between the two integers. This is the reason that after the discovery of fractional calculus, it is observed that FDEs have more real in natural phenomena than to the ODEs [5-6]. As far as the geometrical and physical interpretation of integer order differentials and integrals are concerned, they have the clear physical interpretation in calculus. Fractional derivative and integral are actually the superset of the ordinary or integer order derivative and integral and hence fractional derivative and integral give more deep result and realistic natural phenomena than the ordinary or integer order derivative and integral ones. The geometrical interpretation of Integer order differential and integration can be seen in classical geometry whereas some researchers seek the geometrical interpretation of fractional differential and integral in fractal geometry as the classical geometry is the subclass of fractal geometry $[23,30]$.

The study of FDEs in fractional calculus is one of the most popular subject in many mathematical scientific areas including the image processing, earthquake and biomedical engineering, viscoelasticity [3], finance [25], hydrology [4] and control system [1]. In all these fields of studies, it is important to find out the exact or approximate solutions of FDEs but unfortunately we do not have any method to find the exact solution of such type of FDEs so therefore we use approximation to the exact solution. To find out the exact solution of such type of FDEs and other scientific application is very difficult task in mathematics. The exact solution help us to understand the mechanism and complexity of the problem as compare to the approximate solution [30]. To obtain the exact analytic solutions of FDEs, it is very difficult 
and some time impossible to deal with the complexities computations in these equations. So it is better to look for some useful approximations and numerically techniques such as variation iteration method [9], homotopy perturbation method [27], collection method [16-20], Galerkin method, Laplace transform and Fourier transform methods and other methods $[2,7,8,10,11,12,13,15,22]$.

The DFDEs are the differential equations in which derivative of unknown function at some certain point is given in terms of the values of the function at previous time. The fraction are also called the Time-Delay System or sometime, Dead -Time System. The fractional partial differential equations with delay have attracted a significant attention of the scientists and researchers due to their frequent appearance in the models of actual applications in the last decades. The DFDEs are mostly used in medical sciences, networks, cell biology, control theory, climate models and many others [30]. The delay term in the fractional partial differential equation does not only make more difficult and complicated to the exact solution but also change the long time dynamic property of system. This is the reason that makes researcher to attract such type of equations for simulation.

The DFDEs (Delay Fractional Differential Equations) have been solved by various mathematician and researchers in literature. But the most recent numerical solution of DFDEs are solved by M.A Iqbal el at.[14] (2013) by Chebyshev wavelets numerical technique , M.M Khader and A.S Hendy [16] (2015), used the legendre seudospectral method , N. Mahmoud Sherif el at. [21] (2014) used the spline functions technique, H. Osama Mohammed and Abbas I. Khlaif [24] (2014) used Adomian Decomposition Method (ADM).

The organization of this paper is as follows: In section 2, we introduce some notations and definitions regarding to Caputo's fractional derivatives and delay differential equation of fractional order. In section 3, we introduce Chebyshev polynomials on interval $[-1,1]$ and shifted chebyshev polynomials on the interval $[0,1]$ and find the approximate formula for fractional derivative with weight function. In section 3, we give the procedure for solving DFDE and collocate formulated equation at some suitable points together with boundary values conditions. In section 4 , we present two numerical examples to show the validity of the method. In this section we compare the solution of one of the example with example solved in [24] and find that our solved example give more accuracy to the exact solution than the one's in [24]. Also the graphically comparison between the exact and approximate values by the proposed method are given. Finally in last section, we give some remarks about calculations and graphs in our paper.

\section{Preliminaries and notations}

Definition 1.The Caputo's fractional derivative w.r.t $x$ of order $\alpha$ denoted by $\frac{d^{(\alpha)} f(x)}{d x}$ and defined as follows:

$$
\frac{d^{(\alpha)} f(x)}{d x}=\frac{1}{\Gamma(m-\alpha)} \int_{0}^{x} \frac{f^{(m)}(t)}{(x-t)^{\alpha-m+1}} d t, m-1<\alpha<m, m \in N x>0, \alpha>0
$$

For the function of the type $f(x)=x^{n}, n \in W=\{0,1,2 \ldots\}$.The Caputo's fractional derivative is defined as:

$$
\frac{d^{(\alpha)} x^{2}}{d x}= \begin{cases}0, \quad \text { for } \quad n \in W & n<\lceil\alpha\rceil \\ \frac{\Gamma(n+1)}{\Gamma(n+1-\alpha)} x^{n-\alpha}, \quad \text { for } \quad n \in W \quad n \geq\lceil\alpha\rceil\end{cases}
$$

Here $\lceil\alpha\rceil$ denotes the ceiling value of $\alpha$.It the smallest integer greater than or equal to $\alpha$. Moreover if $f(x)=c$ is any constant function then its the Caputo's fractional derivative will be zero like the integer order derivative i.e $\frac{d^{(\alpha)} c}{d x}=$ 
0. Amongst the main properties of Caputo's fractional derivative, the most important property is the linearity property i.e

$$
\frac{d^{(\alpha)}}{d x}(\lambda f(x)+\mu g(x))=\lambda \frac{d^{(\alpha)}}{d x} f(x)+\mu \frac{d^{(\alpha)}}{d x} g(x)
$$

where $\lambda$ and $\mu$ are constants [25].

In this paper, we use the following type of the delay differential equation of fractional order $\alpha$.

$$
\frac{d^{(\alpha)}}{d x} y(x)=f(x, y(x), y(g(x))), a \leq x \leq b, m<\alpha \leq m+1, m=1,2,3 \ldots
$$

with the following boundary conditions;

$$
y(a)=\beta_{0}, y(b)=\beta_{1}, y(x)=\psi(x), x \in\left[a_{0}, a\right]
$$

If $\alpha=2$,the above equation (1) is called classical second-order delay differential equation. In this case, we have

$$
\frac{d^{2}}{d x} y(x)=f(x, y(x), y(g(x))), a \leq x \leq b
$$

Here $g$ is the delay function which is to be assumed continues in the interval $[a, b]$ and satisfies the inequality $a_{0} \leq g(x) \leq x$ for some fix real constant $a_{0}$ for $x \in[a, b], \psi \in C\left[a_{0}, a\right]$.

\section{Definitions and properties of chebyshev polynomials}

Many authors and researcher use the Chebyshev polynomials not only for the solution of ODEs but also they use for solving the FDEs. The Chebyshev polynomials are a sequence of orthogonal polynomials and widely used in many areas of numerical analysis for simulation like least square approximation, uniform approximation and in spectral or pseudospectral methods. The Chebyshev polynomials are also very important in approximation theory for interpolation. One of the advantages of Chebyshev polynomials is the approximation of a function $f(x)$ by a polynomial $p(x)$ that gives a uniform and accurate description in the real interval $[a, b]$. Another advantage is to approximate a function $f(x)$ in terms of series expansion that form the basis of the FDEs [30]. The recurrence formula for Chebyshev polynomials (also known as Chebyshev polynomials of first kind) on the interval $[1,-1]$ is as follows:

$$
\begin{gathered}
T_{0}(x)=1, T_{1}(x)=x \\
T_{n+1}(x)=2 x T_{n}(x)-T_{n-1}(x), n=1,2 \ldots
\end{gathered}
$$

The orthogonality condition is

$$
\int_{-1}^{1} \frac{T_{i}(x) T_{j}(x)}{\sqrt{1-x^{2}}} d x=\left\{\begin{array}{l}
\Pi, \text { for } i=j=0 \\
\frac{\Pi}{2}, \text { for } i=j \neq 0 \\
0, \text { for } i \neq j
\end{array}\right.
$$

The analytic form of Chebyshev polynomials $T_{n}(x)$ of degree $n$ over the interval $[-1,1]$ is given by ;

$$
T_{n}(x)=n \sum_{i=0}^{\left\lceil\frac{n}{2}\right\rceil}(-1)^{i} 2^{(n-2 i-1)} \frac{(n-i-1) !}{(i) !(n-2 i)} x^{n-2 i}
$$

where $\left\lceil\frac{n}{2}\right\rceil$ denotes the integer part of $\frac{n}{2}$ In order to use these polynomials, it is difficult to work over the interval $[-1,1]$.For easy computations we shift these polynomials on the interval $[0,1]$ and define the so called shifted Chebyshev polynomials 
by introducing the change of variable $x=2 t-1$. The recurrence formula of the shifted Chebyshev polynomials $T_{n}^{*}(t)$ are defined as :

$$
T_{n}^{*}(t)=T_{n}(2 t-1)=T_{2 n}(\sqrt{t})
$$

where

$$
\begin{gathered}
T_{0}^{*}(x)=1, T_{1}^{*}(x)=2 x-1 \\
T_{n+1}^{*}=(4 x-2) T_{n}^{*}-T_{n-1}^{*}, n=1,2,3 \ldots
\end{gathered}
$$

The analytic form of shifted Chebyshev polynomials $T_{n}^{*}(t)$ of degree $n$ is given by ;

$$
T_{n}^{*}(t)=n \sum_{k=0}^{n}(-1)^{n-k} \frac{2^{2 k}(n+k-1) !}{(2 k) !(n-k) !} t^{k}
$$

where $T_{n}^{*}(0)=(-1)^{n}$ and $T_{n}^{*}(1)=1$. The orthogonality conditions for the shifted Chebyshev polynomial are given by,

$$
\int_{0}^{1} \frac{T_{i}^{*}(x) T_{j}^{*}(x)}{\sqrt{x-x^{2}}} d x=\delta_{i, j} h_{j}
$$

where $h_{k}=\frac{b_{k}}{2} \Pi$ with $b_{0}=1, b_{k}=1, k \geq 1$.

We can approximate the solution of the equation of the type defined in (1) with the boundary conditions in (2) as the infinite series of shifted Chebyshev polynomials,

$$
x(t)=\sum_{i=0}^{\infty} c_{i} T_{i}^{*}(t)
$$

The coefficients $c_{i}$ are given by;

$$
c_{i}=\left\{\begin{array}{l}
\frac{1}{\Pi} \int_{0}^{1} \frac{x(t) T_{0}^{*}(t)}{\sqrt{t-t^{2}}} d t, \quad \text { for } i=0 \\
\frac{2}{\Pi} \int_{0}^{1} \frac{x(t) T_{i}^{*}(t)}{\sqrt{t-t^{2}}} d t, \text { for } i=1,2 \ldots
\end{array}\right.
$$

We consider the first $m+1$ terms of the shifted Chebyshev polynomials. So Eq.(4) will take the form :

$$
x_{m}(t)=\sum_{i=0}^{m} c_{i} T_{i}^{*}(t)
$$

Theorem 1.Let $x_{m}(t)$ be the approximate solution of DFDE as defined in Eq.(5) and also suppose that $\alpha>0$, then

$$
\frac{d^{\alpha} x_{m}(t)}{d x}=\sum_{i=\lceil\alpha\rceil k=\lceil\alpha\rceil}^{m} \sum_{i}^{i} \Omega_{i, k}^{(\alpha)} t^{k-\alpha}
$$

where $\Omega_{i, k}^{(\alpha)}$ is weight function and defined as

$$
\Omega_{i, k}^{(\alpha)}=(-1)^{i-k} \frac{2^{2 k} i(i+k-1) ! \Gamma(k+1)}{(i-k) !(2 k) ! \Gamma(k+1-\alpha)}
$$


Proof.Due to linearity property of Caputo's fractional derivative we can write Eq.(5) as

$$
\frac{d^{\alpha} x_{m}(t)}{d x}=\sum_{i=\lceil\alpha\rceil}^{m} c_{i} \frac{d^{\alpha}}{d x}\left(T_{i}^{*}(t)\right)
$$

From definition (1), $\frac{d^{\alpha}}{d x}\left(T_{i}^{*}(t)\right)=0, \forall i=0,1, \ldots\lceil\alpha\rceil-1, \alpha>0$.

Again apply property of linearity on the analytic form of the shifted Chebyshev polynomials described in Eq.(3), we have

$$
\frac{d^{\alpha}}{d x}\left(T_{i}^{*}(t)\right)=i \sum_{k=\lceil\alpha\rceil}^{i}(-1)^{i-k} \frac{2^{2 k}(i+k-1) !}{(i-k) !(2 k) !} \frac{d^{\alpha}}{d x}\left(t^{k}\right) \forall, i=\lceil\alpha\rceil+\lceil\alpha\rceil+1 \ldots . m
$$

Apply definition (1), we have

$$
\frac{d^{\alpha}}{d x}\left(T_{i}^{*}(t)\right)=i \sum_{k=\lceil\alpha\rceil}^{i}(-1)^{i-k} \frac{2^{2 k}(i+k-1) ! \Gamma(k+1)}{(i-k) !(2 k) ! \Gamma(k+1-\alpha)} t^{k-\alpha}
$$

Using values from Eq.(7) into Eq.(6), we have

$$
\frac{d^{\alpha} x_{m}(t)}{d x}=\sum_{i=\lceil\alpha\rceil}^{m} \sum_{k=\lceil\alpha\rceil}^{i} c_{i}(-1)^{i-k} \frac{2^{2 k} i(i+k-1) ! \Gamma(k+1)}{(i-k) !(2 k) ! \Gamma(k+1-\alpha)} t^{k-\alpha}
$$

as desired.

\section{Procedure for solution of the DFDE}

In this section we use the Chebyshev's collocation method to solve the DFDE (1) together with the boundary conditions (2) described in next section. For this purpose suppose that the approximate solution $y(x)$ is in term of finite number $m$ of series of shifted Chebyshev polynomials.i.e

$$
y_{m}(x)=\sum_{i=0}^{m} c_{i} T_{i}^{*}(x)
$$

Employing the Theorem (3) and Eq.(8) into the Eq.(1),we have

$$
\sum_{i=\lceil\alpha\rceil k=\lceil\alpha\rceil}^{m} \sum_{i}^{i} \Omega_{i, k}^{(\alpha)} x^{k-\alpha}=f\left(x, \sum_{i=0}^{m} c_{i} T_{i}^{*}(x), \sum_{i=0}^{m} c_{i} T_{i}^{*}(g(x)), 0<x<1, m+1<\alpha<m\right.
$$

By using the collocation method we collocate the above Eq.(4.2) at the points $x_{p}, p=0,1, \ldots m-\lceil\alpha\rceil$ i.e total number of collocated points will be $(m+1-\lceil\alpha\rceil)$. For appropriate collocated points we use the roots of the shifted Chebyshev polynomial $T_{m+1-\lceil\alpha\rceil}^{*}(x)$.

$$
\sum_{i=\lceil\alpha\rceil k=\lceil\alpha\rceil}^{m} \sum_{i}^{i} \Omega_{i, k}^{(\alpha)} x_{p}^{k-\alpha}=f\left(x_{p}, \sum_{i=0}^{m} c_{i} T_{i}^{*}\left(x_{p}\right), \sum_{i=0}^{m} c_{i} T_{i}^{*}\left(g\left(x_{p}\right)\right), x_{p}, p=0,1, \ldots m-\lceil\alpha\rceil, m+1<\alpha<m\right.
$$

Employing the Eq.(8) in the boundary conditions Eq.(2), we can obtain $\lceil\alpha\rceil$ equations as follows :

$$
\sum_{i=0}^{m}(-1)^{i} c_{i}=\beta_{0}, \sum_{i=0}^{m} c_{i}=\beta_{1}
$$


From Eq.(10) we get $(m+1-\lceil\alpha\rceil)$ algebraic equations and from Eq.(11) we get $\lceil\alpha\rceil$ algebraic equations so we get total $(m+1)$ linear or non-linear algebraic equations which can easily be solved with the help of matrices for unknowns $c_{n}, n=0,1,2 \ldots m$ to calculate the approximate solution $y_{m}(x)$.

\section{Numerical implementation}

In this section, we solve numerically the fractional order delay differential equations using properties of shifted Chebyshev polynomials.All the numerical results are obtained using MATLAB.

Example 1. Consider the following linear fractional delay differential equation ;

$$
D^{1.5} u(x)=-u(x)+u\left(\frac{x}{2}\right)+\frac{7}{8} x^{3}+\frac{6}{\Gamma(2.5)} x^{1.5}
$$

with the boundary conditions ;

$$
u(0)=0, u(1)=1
$$

The exact solution of this problem is $u(x)=x^{3}$. We implement the suggested method with $m=3$ and $m=5$ we approximate the solution as, let $m=3$,

$$
u_{3}(t)=\sum_{i=0}^{3} c_{i} T_{i}^{*}(x)
$$

Using Eq.(10) we have ,

$$
\sum_{i=2}^{3} \sum_{k=2}^{i} c_{i} \Omega_{i, k}^{(\alpha)} x_{p}^{k-\alpha}=-\sum_{i=0}^{3} c_{i} T_{i}^{*}\left(x_{p}\right)+\sum_{i=0}^{3} c_{i} T_{i}^{*}\left(\frac{x_{p}}{2}\right)+\frac{7}{8} x_{p}^{3}+\frac{6}{\Gamma(2.5)} x_{p}^{1.5}
$$

With $p=0,1$ where $x_{p}$ are the roots of shifted Chebyshev polynomial $T_{2}^{*}(x)$ and their values are:

$$
x_{0}=0.8536, x_{1}=0.1464
$$

By using Eq.(11) and Eq.(12) we have ,

$$
\begin{aligned}
& c_{0}-c_{1}+c_{2}-c_{3}=0 \\
& c_{0}+c_{1}+c_{2}+c_{3}=1
\end{aligned}
$$

Solving the Eq.(13) together with the Eq.(14) and Eq .(15) we find the approximate values $u_{a p x}$ in table (1) for $m=3$ and $m=5$.

Example 2. Consider the following linear fractional delay differential equation ;

$$
D^{\alpha} u(x)=\frac{3}{4} u(x)+u\left(\frac{x}{2}\right)-x^{2}+2,0 \leq x \leq 1,1<\alpha \leq 2
$$

with the boundary conditions:

$$
u(0)=0, u(1)=1
$$

The exact solution of this problem is $u(x)=x^{2}$. We implement the suggested method with $m=3$ and $\alpha=1.5,1.75,2$ as follows,

$$
u_{3}(t)=\sum_{i=0}^{3} c_{i} T_{i}^{*}(x)
$$


Table 1: Comparison of exact and approximate values at $m=3$ and $m=5$ with $\alpha=1.5$

\begin{tabular}{|c|c|c|c|}
\hline$x$ & $u_{\text {ext }}$ & Error $=\left|u_{\text {ext }}-u_{\text {apx }}\right|$ at $m=3$ & Error $=\left|u_{\text {ext }}-u_{\text {apx }}\right|$ at $m=5$ \\
\hline 0.0000 & 0.0000 & $6.9389 e^{-18}$ & $6.9389 e^{-18}$ \\
\hline 0.1000 & 0.0010 & $5.7600 e^{-5}$ & $5.7600 e^{-5}$ \\
\hline 0.2000 & 0.0080 & $7.6800 e^{-5}$ & $7.6800 e^{-5}$ \\
\hline 0.3000 & 0.0270 & $6.7200 e^{-5}$ & $6.7200 e^{-5}$ \\
\hline 0.4000 & 0.0640 & $3.8400 e^{-5}$ & $3.8400 e^{-5}$ \\
\hline 0.5000 & 0.1250 & $0.0000 e^{-0}$ & $0.0000 e^{-0}$ \\
\hline 0.6000 & 0.2160 & $3.8400 e^{-5}$ & $3.8400 e^{-5}$ \\
\hline 0.7000 & 0.3430 & $6.7200 e^{-5}$ & $6.7200 e^{-5}$ \\
\hline 0.8000 & 0.5120 & $7.6800 e^{-5}$ & $7.6800 e^{-5}$ \\
\hline 0.9000 & 0.7290 & $5.7600 e^{-5}$ & $5.7600 e^{-5}$ \\
\hline 1.0000 & 1.0000 & $0.0000 e^{-0}$ & $0.0000 e^{-0}$ \\
\hline
\end{tabular}

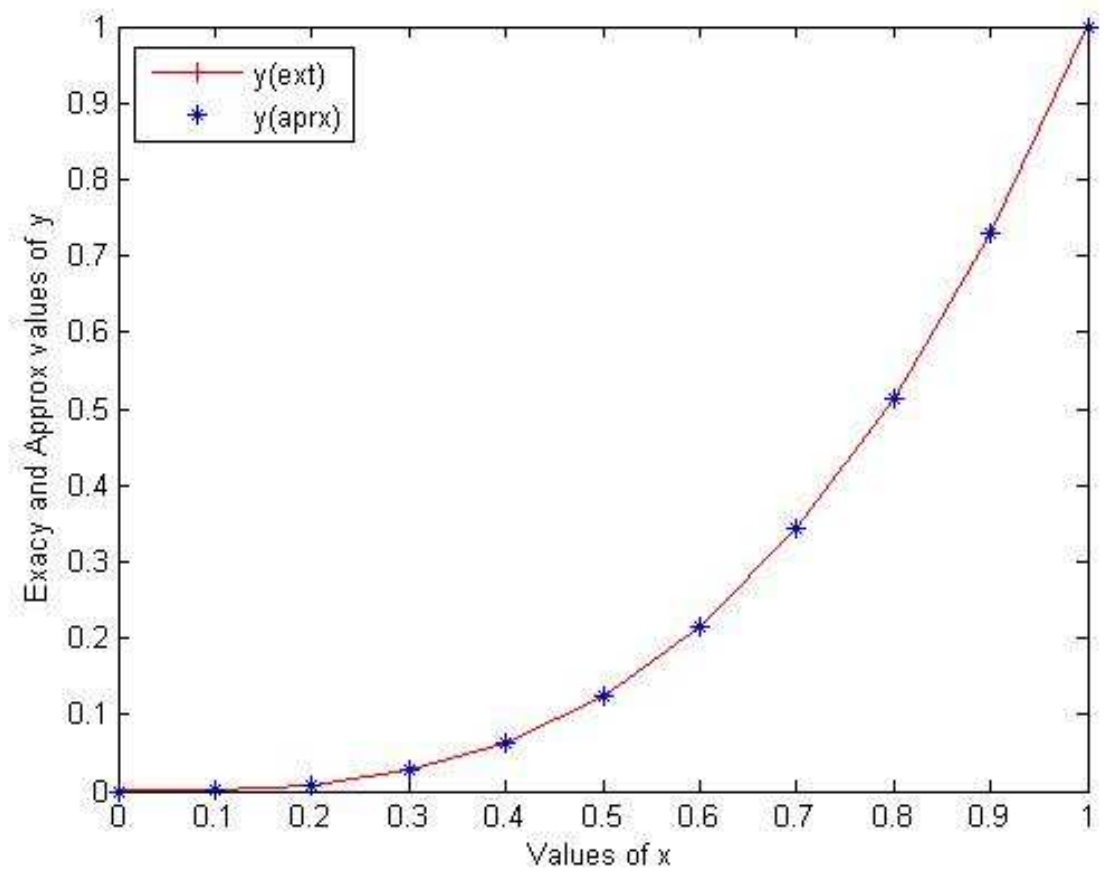

Fig. 1: Result of example 1 by present method taking $m=3, m=5$ and $\alpha=1.5$.

Using Eq.(10) we have

$$
\sum_{i=2}^{3} \sum_{k=2}^{i} c_{i} \Omega_{i, k}^{(\alpha)} x_{p}^{k-\alpha}=\frac{3}{4} \sum_{i=0}^{3} c_{i} T_{i}^{*}\left(x_{p}\right)+\sum_{i=0}^{3} c_{i} T_{i}^{*}\left(\frac{x_{p}}{2}\right)+x_{p}^{2}+2
$$

With $p=0,1$ where $x_{p}$ are the roots of shifted Chebyshev polynomial $T_{2}^{*}(x)$ and their values are

$$
x_{0}=0.8536, x_{1}=0.1464
$$

By using Eq.(4) and Eq.(2) we have,

$$
c_{0}-c_{1}+c_{2}-c_{3}=0
$$




$$
c_{0}+c_{1}+c_{2}+c_{3}=1
$$

Solving the Eq.(14) together with the Eq.(15) and Eq.(16) we find the approximate values $u_{a p x}$ for different values of $\alpha$ in table (2) for $m=3$ with comparison of the estimated values calculated in [24] by ADM.

Table 2: Comparison of exact and approximate values at $\alpha=1.5,1.75,2$ and $m=3$ with Ref [24]

\begin{tabular}{|c|c|c|c|c|c|c|c|}
\hline$x$ & Exact & $\operatorname{Ref}[24] \alpha=1.5$ & ours $\alpha=1.5$ & $\operatorname{Ref}[24] \alpha=1.75$ & ours $\alpha=1.75$ & $\operatorname{Ref}[24] \alpha=2$ & ours $\alpha=2$ \\
\hline 0.0000 & 0.0000 & 0.0000 & 0.0000 & 0.0000 & 0.0000 & 0.0000 & 0.0014 \\
\hline 0.1000 & 0.0100 & 0.0480 & -0.03460 & 0.0220 & -0.0095 & 0.0100 & 0.0129 \\
\hline 0.2000 & 0.0400 & 0.1370 & -0.0247 & 0.0750 & 0.0102 & 0.0400 & 0.0444 \\
\hline 0.3000 & 0.0900 & 0.2550 & 0.0241 & 0.1530 & 0.0572 & 0.0900 & 0.0957 \\
\hline 0.4000 & 0.1600 & 0.3980 & 0.1064 & 0.2540 & 0.1297 & 0.1600 & 0.1667 \\
\hline 0.5000 & 0.2500 & 0.5640 & 0.2168 & 0.3770 & 0.2260 & 0.2500 & 0.2572 \\
\hline 0.6000 & 0.3600 & 0.7530 & 0.3499 & 0.5210 & 0.3442 & 0.3600 & 0.3672 \\
\hline 0.7000 & 0.4900 & 0.9630 & 0.5001 & 0.6870 & 0.4825 & 0.4900 & 0.4964 \\
\hline 0.8000 & 0.6400 & 1.1950 & 0.6622 & 0.8730 & 0.6391 & 0.6400 & 0.6448 \\
\hline 0.9000 & 0.8100 & 1.1448 & 0.8306 & 1.0800 & 0.8122 & 0.8090 & 0.8123 \\
\hline 1.0000 & 1.0000 & 1.7230 & 1.0000 & 1.3070 & 1.0000 & 0.9990 & 0.9986 \\
\hline
\end{tabular}

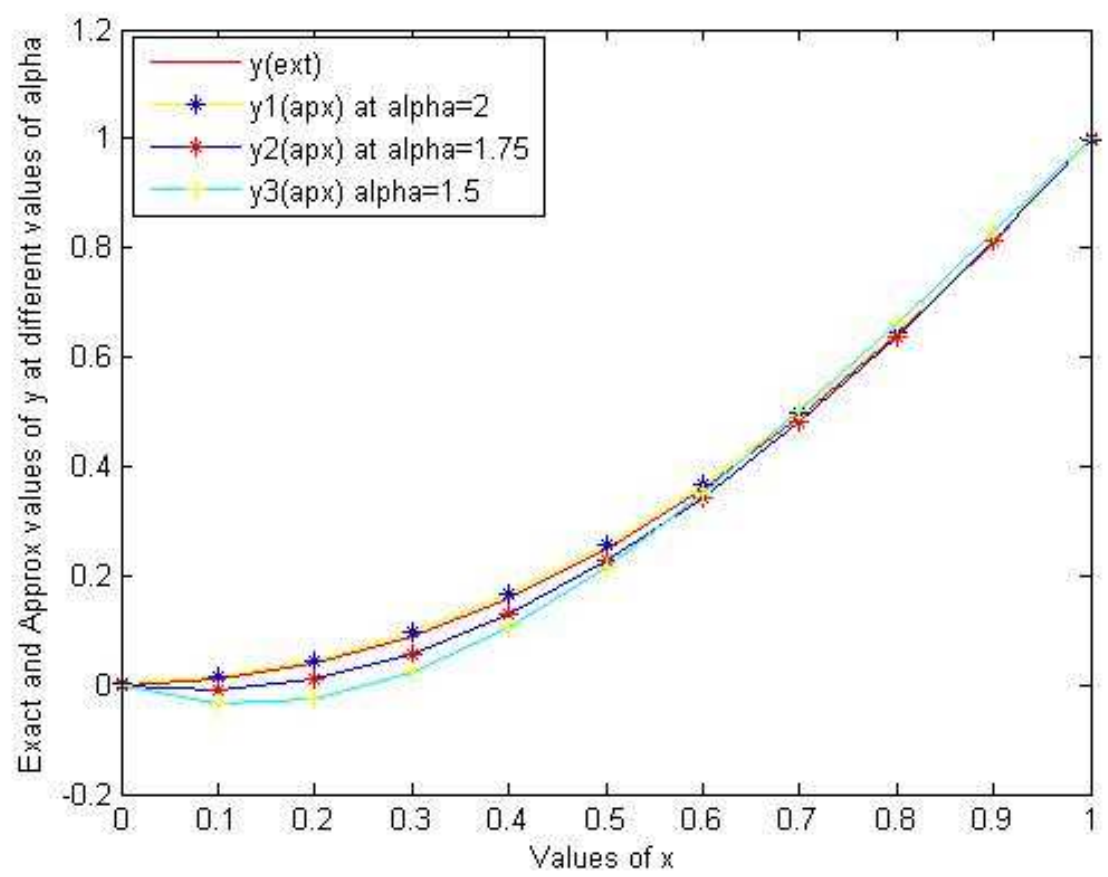

Fig. 2: Result of example 2 by present method taking $m=3$ and $\alpha=1.5,1.75,2$. 


\section{Conclusion and remarks}

In this paper, we successfully applied Chebyshev collocation method to solve the delay differential equations of fractional order. For fractional differential,we use Caputo's fractional derivatives. The properties of Chebyshev polynomials are utilized to reduce DFDEs into easily solvable linear or nonlinear algebraic equations. Two numerically solved examples show the present method is well organized.The calculated approximate values are in excellent agreement with the exact solutions and hence this approach can solve the problem efficiently. In our suggested method, the calculated estimate values is much more accurate than the values calculated by (ADM) in [24]. One can easily see the difference in both methods with the help of the graph of approximate values at different values of $\alpha$. This shows that our suggested method is more effective, valid and applicable.

\section{Competing interests}

The authors declare that they have no competing interests.

\section{Authors' contributions}

All authors have contributed to all parts of the article. All authors read and approved the final manuscript.

\section{References}

[1] O. P. Agrawal, O. Defterli and D. Baleanu, Fractional optimal control problems with several state and control variables, Journal of Vibration and Control, vol. 16, no. 13, pp. 1967-1976, (2010).

[2] A. M. Akinlar, Aydin Secer and Mustafa Bayram, Numerical Solution of fractional Benney equation, Appl. Math. Inf. Sci. 8, No. 4, pp. 1633-1637, (2014).

[3] R. L. Bagley and P. J. Torvik, Theoretical basis for the application of fractional calculus to viscoelasticity, Journal of Rheology, Vol. 27, No. pp.201-210, (2013).

[4] D. A. Benson and S. W. Wheatcraft, Application of a fractional advection-dispersion equation, Water Resources Research, vol. 36, No. 6, pp. 1403-1412, (2000).

[5] C. M. Chen, F. Liu, V. Anh and I. Turner, Numerical methods for solving a two dimensional variable-order anomalous subdiffusion equations, Mathematics of Computation Vol. 81, No. 277, pp. 345-366, (2012).

[6] A. Concepcion Monje, Yangquan Chen, M.Vinangre, Dingyu Xue, Vicente Feliu, Fractional order systems and controls fundamentals and applications, Adva. Indus. Con. Springer-Verlag, London, (2010).

[7] H. D. Eid , Ali H. Bhrawy, Dumitru Baleanu and Samer S. Ezz-Eldien, The operational matrix formulation of the Jacobi tau approximation for space fractional diffusion equation, Springer open journal, Doha et al. Advances in difference equations, pp. 201-231, (2014).

[8] I. I. Gorial, Numerical Solution for fractional Partial Differential euations of two sided, Journal of Al-Nahrain University Vol. 12 (2), , pp. 128-131, (2009).

[9] J. He, Approximate analytical solution for fractional derivatives in porous media, Computer Methods in Applied Mechanics and Engineering, 167 (1-2), pp. 57-68, (1998).

[10] A. A. Hemeda, Homotopy Perturbation Method for Solving Partial Differential Equations of Fractional Order, Int. Journal of Math. Analysis, Vol. 6, No. 49, pp.2431 - 2448,(2012).

[11] K. Ibrahim, Nurdane Kale, Serife R. Bayramoglu, A New Difference Scheme for time fractional Heat equations based on the crank-nicholson method, An Int. J. for Theory and Applications, Vol. 16, No. 4 (2013).

[12] K. Ibrahim, Nurdane Kale, Finite difference method of fractional parabolic partial differential equations with variable coefficients, Int J. of Contemporary Mathematics Sciences, Vol.9, No. 16, pp. 767-776, (2014). 
[13] K. Ibrahim, Nurdane Kale, Serife R. Bayramoglu, A characheristics difference scheme for time fractional heat equations based on the crank-nicholson difference scheme, Hindawi publishing corporation Abstract and Applied Analysis Vol. 2012, Article ID 548292, doi:10.1155/2012/548292.

[14] M. A Iqbal, Ayyaz Ali and S. T. Mohyud-Din, Chebyshev Wavelets Method for Fractional Delay Differential Equations, International Journal of Modern Applied Physics, 4(1), pp. 49-61, (2013).

[15] H. Jafari, M. Soleymanivaraki, M. A. Firoozjaee, Legendre Wavelets for Solving Fractional Differential Equations, Journal of Applied Mathematics, Vol. 7, No. 4(27), pp. 65-70, (2011).

[16] M. M. Khader, A. S Hendy, The approximate and exact solutions of the fractional-order delay differential equations using legendre seudospectral method, Int J. Pure and Applied Mathematics, Vol. 74 No. 3 pp. 287-297, (2012).

[17] M. M. Khader, Numerical solution of Nonlinear Multi-order fractional differential equations by implementation of the operational derivative, Studies in Nonlinear Sciences 2(1), pp. 05-12, (2011).

[18] M. M. Khadar, N. H. Sweilam, T. A Assiri, On numerical solution for the fractional wave equation using legendre psedospectral method, Int J. Pure and Applied Mathematics, Vol. 84 No. 4 pp. 307-319, (2013).

[19] M. M. Khadar, N. H. Sweilam, A. M. S. Mahdy, An Efficient method for solving the fractional diffusion equation, Int J. Pure and Applied Mathematics, Vol. 1, No. 2, pp. 1-12, (2011).

[20] M. M. Khader, On the numerical solutions for the fractional diffusion equation, Communications in Nonlinear Science and Numerical Simulation, doi: 10.1016/j.cnsns.2010.09.007.

[21] N. Mahmoud Sherif, Ibrahim Abouelfarag, T. S. Amer,Numerical solution of fractional Delay dufferential euations using spline functions, International Journal of Pure and Applied Mathematics, Vol. 90, pp. 73-83, (2014).

[22] M. M. Meerschaert, H. P. Scheffler, C. Tadjeran, Finite difference methods for two-dimensional fractional dispersion equation, Journal of Computational Physics pp. 249-261, (2006).

[23] Moshrefi, Hammond, Physical and geometrical interpretations of fractional operators, Elsevier science, (1998).

[24] H. Osama Mohammed and Abbas I. Khlaif, Adomian Decomposition Method for Solving Delay Differential Equations of Fractional Order, IOSR Journal of Mathematics, Vol. 10, Issue 6 Ver. I, pp. 1-5, (2014).

[25] I.Podlubny, Fractional Differential Equations, Academic Press, New York, (1999).

[26] E. Scalas, R. Gorenflo, and F. Mainardi, Fractional calculus and continuous-time finance, Physica A, Vol. 284, No. 1-4, pp. 376384, (2000).

[27] M. Seifollahi, A. S Shamloo, Numerical solution of non linear muti-order fractional differential equations by operational matrix of chebyshev polynomials, World Applied Programming Vol. (3), pp. 85-92, (2013).

[28] N. M. Sweilam, M. M. Khader and R. F. Al-Bar, Homotopy perturbation method for multidimensional nonlinear coupled system of parabolic and hyperbolic equations, Topological Methods in Nonlinear Analysis, Vol 31, pp. 295-304.

[29] G. Wanhai, Yiming Chen, Wavelet Method for Nonlinear Partial Differential Equations of Fractional Order, Computer and Information Science, Vol. 4, No. 5, doi:10.5539/cis.v4n5p28, (2011).

[30] J. Wu, Theory and Applications of Partial Functional Differential Equations, New York, NY, USA, Springer, (1996). 